\title{
Direct and maternal reduced balanced protein diet influences the liver transcriptome in chickens
}

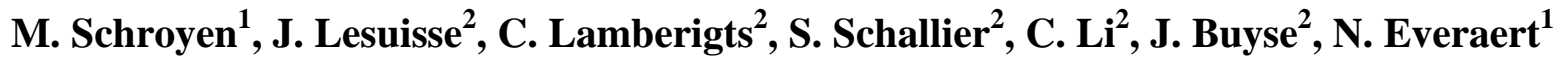 \\ ${ }^{1}$ Precision Livestock and Nutrition Laboratory, Teaching and Research Centre (TERRA), \\ Gembloux AgroBioTech, University of Liège, Passage des Déportés 2, 5030 Gembloux, \\ Belgium. \\ ${ }^{2}$ Laboratory of Livestock Physiology, Department of Biosystems, KU Leuven, Kasteelpark \\ Arenberg 30, 3001 Heverlee, Belgium.
}

Corresponding author: Johan Buyse, Laboratory of Livestock Physiology, Department of Biosystems, KU Leuven, Kasteelpark Arenberg 30, 3001 Heverlee, Belgium, Johan.Buyse@kuleuven.be, tel: +32 163285 25, fax: +32 16321994.

Short title ( $\max 45$ characters): Metabolism affected by maternal protein diet

Keywords: broiler breeder chickens, reduced protein diet, liver transcriptome, match-mismatch diets

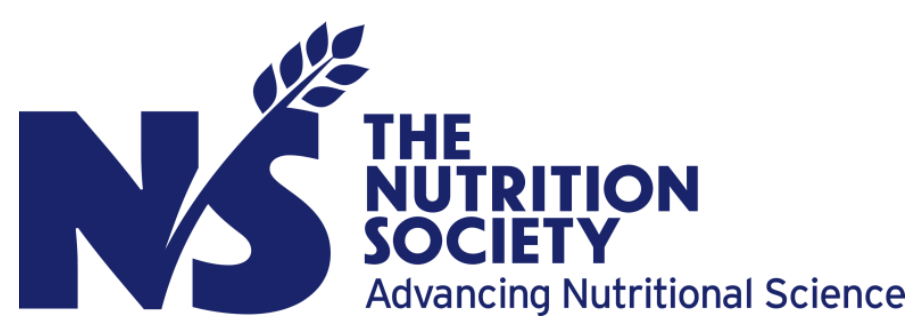

This peer-reviewed article has been accepted for publication but not yet copyedited or typeset, and so may be subject to change during the production process. The article is considered published and may be cited using its DOI 10.1017/S0007114520003785

The British Journal of Nutrition is published by Cambridge University Press on behalf of The Nutrition Society 


\section{Abstract (max 250 words)}

The objective of this study was to evaluate, by means of RNA-sequencing, the direct and transgenerational effect of a reduced balanced protein diet on broiler breeder metabolism. Chickens of the F0 generation were fed a control (C) or reduced balanced protein (RP) diet and their F1 progeny was fed a $\mathrm{C}$ or RP diet as well, resulting in four groups of chickens, $\mathrm{C} / \mathrm{C}, \mathrm{C} / \mathrm{RP}$, RP/C and RP/RP. While both a direct and a maternal effect were seen on body weight, breast muscle weight and abdominal fat weight in the F1 generation, the direct effect was the most dominant one. The liver transcriptome in the F1 generation showed that amino acid metabolism was upregulated in chickens that received control feed when compared to their respective contemporaries that received the reduced protein diet. Interestingly, chickens hatched from control fed hens but reared on reduced protein diet (C/RP group) activated a fatty acid metabolism, expressing more FADS1, FADS2 and ELOVL2, when compared to control fed chickens hatched from control fed hens ( $\mathrm{C} / \mathrm{C}$ group), while chickens hatched from reduced protein fed hens that received themselves the same reduced protein diet (RP/RP group) triggered their glucose metabolism more, showing elevated levels of PFKL, PFKFB4 and ALDOC mRNA compared to the chickens hatched from reduced protein fed hens but reared on a control diet (RP/C group). This suggests that the maternal protein diet has an impact on the metabolism of broilers when they are reared on a reduced balanced protein diet. 


\section{Introduction}

Over the last decade, reducing dietary protein has been of great interest to the poultry industry. Lowering crude protein in broiler feed can decrease nitrogen emissions and hence diminish the negative impact on the environment ${ }^{(1)}$. Additionally, protein is one of the most expensive components of poultry diets, especially in Europe, and reducing it is therefore an effective way to lower production costs providing the animal's performance is not negatively affected ${ }^{(2,3)}$. However, not only the animal's own performance should be validated since protein content and protein source in the maternal diet are also important when considering pre- and postnatal development $^{(4)}$. Maternal programming during early-life developmental stages has been shown to affect the progeny in the long term. Plenty of research focuses on the influence of maternal dietary changes during gestation and/or lactation on the health status and performance of the progeny. In broiler chickens, maternal diets have been seen to affect offspring's body weight and carcass yield $^{(5)}$, growth ${ }^{(6-8)}$, lipid metabolism ${ }^{(6,9,10)}$, feeding behavior and neophobic attitude ${ }^{(11,12)}$. In the current study, a maternal diet based on reduced crude protein levels in female broiler breeder chickens is investigated. A two-by-two experimental design, in which female broiler breeders as well as their progeny received a reduced balanced protein diet or an isoenergetic control regime, enables to investigate a match (similar diet) or mismatch (dissimilar diet) between mother and offspring nutritional environment. A mismatch is known to result in the suboptimal development of the offspring ${ }^{(13)}$. The question raised here was if chickens, when fed a reduced protein diet while descending from mothers fed a lower amount of protein (match), could have altered their liver metabolism in such a way that their performance increased compared to than those reared on a reduced protein diet but born from mothers that received a normal amount of protein (mismatch). Along the same reasoning, it was investigated if there was a difference in control fed animals, descending from control fed mothers (match) rather than reduced protein fed mothers (mismatch).

\section{Material and Methods}

\section{Animals}

In this project, the maternal influence of a low protein diet was investigated in broiler breeder chickens. To this extent, a control (C) or reduced balanced protein (RP) diet was fed to two generations of chickens: while the F0 generation consisted of two groups ( $\mathrm{C}$ and RP), the F1 
generation contained 4 groups in total, $\mathrm{C} / \mathrm{C}, \mathrm{C} / \mathrm{RP}, \mathrm{RP} / \mathrm{C}$ and $\mathrm{RP} / \mathrm{RP}$, with letters indicating the breeder diet from 3 weeks of age till slaughtering in respectively the F0 and F1 generation. More information on growth and reproductive performance of the $\mathrm{C}$ versus RP birds of the F0 generation can be found in Lesuisse et al. ${ }^{(14)}$. For the F1 generation, more details on their performance parameters and that of their offspring have also been described earlier ${ }^{(15,16)}$. In addition, Lesuisse et al. ${ }^{(15)}$ gives a detailed description of the dietary treatments administered to the chickens discussed in this article. In short, RP fed chickens received a feed with a $25 \%$ reduction in dietary crude protein and amino acids. Feed allocations were however weekly adapted to maintain a similar body weight for all groups throughout the entire experiment ${ }^{(17)}$. In the rearing period, at 10 weeks of age, this resulted in a $13 \%$ higher feed allocation on average and consequently, in a $15 \%$ reduction in daily protein intake in the RP fed chickens compared to the $\mathrm{C}$ fed chickens when grown to the same body weight. In the laying period, there was an average net protein intake reduction of $6 \%$ in the RP/C, of $15 \%$ in $\mathrm{C} / \mathrm{RP}$ and of $17 \%$ in RP/RP chickens compared to $\mathrm{C} / \mathrm{C}$ chickens ${ }^{(15)}$. All animal procedures were approved by the Animal Ethics Committee of the KU Leuven (accession $n^{\circ} \mathrm{P} 187 / 2013$ ).

\section{Sampling and data collection}

Twenty female chickens of the F1 generation, 5 per dietary group, were euthanized by electrical stunning prior to decapitation before feeding at 44 weeks of age. Of these chickens, as described previously, several body composition parameters such as total weight and weight of liver, breast muscle (BM), adipose fat pad (AF) and pancreas were measured ${ }^{(15)}$. The proportional tissue weights were calculated by dividing the absolute tissue weight by the liver weight of the corresponding breeder. Liver samples of all chickens were collected, snap-frozen in liquid nitrogen and stored at $-80^{\circ} \mathrm{C}$ until further RNA processing. Five animals per dietary group were chosen, which surmounts to the number seen in other liver RNA-seq studies to give significant results $^{(18,19)}$.

\section{Statistics on performance parameters}

Body composition parameters were analysed with the statistical software SAS University (SAS Institute Inc., Cary, NC) using a GLM with maternal dietary treatment of the F0 generation,dietary treatment of the F1 generation and their interaction as classification variables. 
When there was a significant effect of any of the dietary treatments or their interaction, means were further compared by a post-hoc Tukey's test. Data are presented as mean \pm SEM in Supplementary Table 1. For each result, the significance level was set at $\mathrm{p}<0.05$.

RNA extraction, library construction and RNA-seq processing

Total RNA was extracted from liver samples of the 20 female F1 chickens using TRIzol ${ }^{\circledR}$ Reagent (Life Technologies, USA) according to the manufacturer's protocol. RNA concentrations were quantified using a NanoDrop ND-1000 spectrophotometer (Nano-Drop Technologies, USA) and quality was assessed using an Agilent Bioanalyzer 2100 (Agilent Technologies, Inc., USA). RIN values were at least 8.5 before further processing. Libraries were constructed using the Illumina TruSeq stranded mRNA kit. RNA-sequencing was performed at the Genomics Core Leuven, Belgium. 125bp paired-end sequencing was performed using the Illumina HiSeq ${ }^{\mathrm{TM}} 2500$ platform. Reads were aligned to the Gallus gallus-5.0 reference genome, Ensembl release 86. Quality of the reads was checked using FASTQC ${ }^{(20)}$ and mapping was done using TopHat ${ }^{(21)}$. All RNA-seq FASTQ files were submitted to the European Nucleotide Archive (ENA) database under accession number PRJEB35156.

\section{RNA-seq differential expression analyses}

The R package DESeq2 was used to investigate differences between experimental groups ${ }^{(22)}$. PCA analyses were performed using the plotPCA function within the DESeq2 package. Subsequently, low reads were filtered out so that the remaining datasets contained solely genes with at least 10 counts in $90 \%$ of the samples, which resulted in 14017 genes. To find differentially expressed genes the Benjamini-Hochberg correction for multiple testing was taken into account $(\mathrm{FDR}<0.05)$. To analyze the differences in function of the genes that were differential expressed (DE), data was corrected for body weight and several comparisons were made. This correction for body weight was done as we aimed to maintain the same body weight in all groups. However, near the end of the experiment, significant differences in body weight were seen due to diet, which prompted the decision to correct the data accordingly. First, we compared groups coming from a similar F0 feed, but a different F1 feed (C/C vs C/RP and RP/C vs RP/RP). Second, analyses were performed keeping the F1 feed constant and changing the F0 feed (C/C vs RP/C and C/RP vs RP/RP). Venny was used to visualize the differences in numbers 
of DE genes between the different comparisons ${ }^{(23)}$. Gene function pathway analyses were performed using the gene ontology (GO) tool $\mathrm{g}$ :profiler ${ }^{(24)}$ with the Ensembl gene ID for Gallus gallus as input and all other options as default. Gene ontology was assessed at the molecular function (MF), biological process (BP) and cellular component (CC) level, and for biological pathways, the KEGG and Reactome were examined.

\section{Weighted Gene Correlation Network Analysis (WGCNA)}

For the comparison of $\mathrm{C} / \mathrm{RP}$ versus RP/RP, a weighted gene co-expression network analysis (WGCNA) was performed to cluster highly correlated genes and to find modules whose expression was significantly correlated with these two groups ${ }^{(25)}$. For the correlations C/RP was set to $0, \mathrm{RP} / \mathrm{RP}$ was set to 1 . Modules were forced to have a minimum of 250 genes and were considered interesting when the nominal p-value for their correlation with the treatment was $\mathrm{p}<$ 0.001. Gene function pathway analyses were also performed on the significant modules as described above.

\section{Results}

\section{Body composition}

Even though it was attempted to keep chickens at the same body weight during the entire experiment, this parameter seemed to be affected by both the F0 and F1 dietary treatment at 44 weeks of age, without interaction effect (Supplementary Table 1). The same could be observed for proportional breast muscle (BM) and proportional abdominal fat pad (AF) (Supplementary Table 1). The proportional liver weight was significantly higher in chickens receiving the RP diet compared to those that received the $\mathrm{C}$ diet $(\mathrm{p}=0.01)$ (Supplementary Table 1). This corresponds to the findings of Lesuisse et al. ${ }^{(15)}$, where the same differences in proportional weight were observed when 5 additional animals per group were measured to the 5 animals in this project.

\section{Differential expression}

The PCA plot revealed $49 \%$ of variance explained on the X-axis which separated the 4 groups mostly according to maternal diet (Figure 1). To analyze the transcriptomic difference between groups of interest, several comparisons were made. First, differences were examined between groups with the same F0 feed, but a different F1 feed (groups C/C vs C/RP and groups RP/C vs 
$\mathrm{RP} / \mathrm{RP}$ ) (Figure 2). This resulted in 91 upregulated genes and 181 downregulated genes in $\mathrm{C} / \mathrm{C}$ compared to $\mathrm{C} / \mathrm{RP}$ and 19 upregulated genes and 32 downregulated genes in $\mathrm{RP} / \mathrm{C}$ compared to the RP/RP group. When comparing the DE in these respective comparisons, 3 genes were found in common (Figure 3, Table 1). Second, groups reared on the same F1 feed, but with a different maternal F0 feed were analysed (groups $\mathrm{C} / \mathrm{C}$ vs $\mathrm{RP} / \mathrm{C}$ and groups $\mathrm{C} / \mathrm{RP}$ vs $\mathrm{RP} / \mathrm{RP}$ ) (Figure 2). This resulted in 22 upregulated genes and 24 downregulated genes in $\mathrm{C} / \mathrm{C}$ compared to $\mathrm{RP} / \mathrm{C}$ and 765 upregulated genes and 1109 downregulated genes in C/RP compared to the RP/RP group. The intersection between these two comparisons comprises 6 genes (Figure 3, Table 1). Looking at all DE genes, $87.1 \%$ of them were found in the comparison between $\mathrm{C} / \mathrm{RP}$ and RP/RP, which is reflected by the division seen by the PCA plot. Two RP/C samples clustered with the C/RP and $\mathrm{C} / \mathrm{C}$ samples in the PCA, and analyses were repeated without these two RP/C samples. This way, more DE genes were found when comparing $\mathrm{C} / \mathrm{C}$ to $\mathrm{RP} / \mathrm{C}$, while less $\mathrm{DE}$ genes were found in the RP/C vs RP/RP comparison, which agrees with the PCA plot. Indeed, a total of 422 genes were upregulated in the $\mathrm{C} / \mathrm{C}$ group when compared with the RP/C group, and 48 genes were upregulated in the $\mathrm{RP} / \mathrm{C}$ when compared to the $\mathrm{C} / \mathrm{C}$ group. When comparing the $\mathrm{RP} / \mathrm{C}$ group with the RP/RP group, only 4 genes were upregulated in the RP/C group, as opposed to 8 upregulated genes in the RP/RP group.

\section{Weighted Gene Correlation Network Analysis (WGCNA)}

Looking at both PCA and DE analyses, the largest difference between groups was found between the $\mathrm{C} / \mathrm{RP}$ and RP/RP chickens. Therefore, additionally, WGCNA was performed on this comparison to identify the hub genes driving this difference. One module was significantly correlated with the groups when coded as 0 and 1 with a correlation coefficient of -0.88 , at a nominal p-value of <0.001. This module contained 1495 genes, whose ENSEMBL gene ID, Gene Significance (GS) and Module Membership (MM) can be found in Supplementary Table 2. Hub genes of the module are those with a high GS and MM value in this table with the gene encoding for methyltransferase like 21C (METTL21C) as top network hub gene.

\section{Functional analyses}

When looking at the direct influence of a protein diet when the maternal diet was a control diet, Gene Ontology analysis by comparing the $\mathrm{C} / \mathrm{C}$ with the $\mathrm{C} / \mathrm{RP}$ group revealed an upregulation of the alpha-amino acid metabolic processes and transaminase activity in the control group (C/C) 
while for the chickens that received the reduced balanced protein diet, the fatty acid metabolism was upregulated (C/RP). When comparing the two different F1 diets in chickens of which the maternal diet was the reduced balanced protein diet, the control diet again showed an upregulation of transaminase activity $(\mathrm{RP} / \mathrm{C})$, while the reduced balanced protein diet now triggered the glucose metabolism (RP/RP). The gene $O A T$, which translates into ornithine aminotransferase, was upregulated in both the $\mathrm{C} / \mathrm{C}$ and $\mathrm{RP} / \mathrm{C}$ group when compared to $\mathrm{C} / \mathrm{RP}$ and $\mathrm{RP} / \mathrm{RP}$ respectively. The expression of this gene was found to be 2.2 fold upregulated in the $\mathrm{C} / \mathrm{C}$ group compared to the $\mathrm{C} / \mathrm{RP}$ group and more than 2.5 fold upregulated in the $\mathrm{RP} / \mathrm{C}$ group compared to the RP/RP group. One of the few upregulated genes C/RP and RP/RP held in common compared to the $\mathrm{C} / \mathrm{C}$ and $\mathrm{RP} / \mathrm{C}$ group respectively, was the gene encoding for teneurin transmembrane protein 2 (TENM2). The upregulated fatty acid metabolism in the C/RP group was reflected by an increase in expression of fatty acid desaturases encoded by the fatty acid desaturase 1 gene (FADS1) and the fatty acid desaturase 2 gene (FADS2) and elongases such as encoded by the elongation of very long chain fatty acids protein 2 gene (ELOVL2). Elevated levels of the phosphofructokinase gene $(P F K L)$, 6-phosphofructo-2-kinase/fructose-2,6biphospatase 4 (PFKFB4) and fructose-biphosphate aldolase $\mathrm{C}$ (ALDOC) were seen in the $\mathrm{RP} / \mathrm{RP}$ group compared to the RP/C group, which points to an activated glucose metabolism. The GO terms differences in the group comparisons remained unaltered when the analysis was done without the two RP/C samples that did not cluster with the other RP F0 diet samples, with again an upregulation of transaminase activity in the RP/C group and an upregulation of protein arginine methyltransferase in the RP/RP group, which indeed has been described to play a role in glucose metabolism ${ }^{(26)}$.

Comparing chickens that received the reduced balanced protein diet but were hatched from hens with a different F0 diet exposed (C/RP vs RP/RP) a nucleocytoplasmic carrier activity elicited by the control maternal diet, while the maternal reduced balanced protein diet showed an upregulation of terms such as 'negative regulation of gene expression'. No informative GO terms appeared to be enriched between the chickens that received the control diet but were hatched from hens with a different diet, not with nor without the two RP/C samples that clustered differently. Raising the threshold for the FDR to 0.10, did not affect the GO terms either, not with all samples, nor without the two RP/C outliers. An overview of the major GO annotations in these comparisons and their enrichment scores are shown in Figure 4. Genes in the significant 
module that correlated with the $\mathrm{C} / \mathrm{RP}$ vs $\mathrm{RP} / \mathrm{RP}$ group were also analysed for $\mathrm{GO}$ annotation and 'phosphotyrosine residue binding' and 'protein phosphorylated amino acid binding' came out as the top GO terms for molecular function. The term 'autophagy' came out as significant KEGG pathway related to this module. A full overview with all genes belonging to the respective GO terms can be found in Supplementary Table 3.

\section{Discussion}

The objective of this study was to examine if maternal programming occurred in the liver of breeder broiler chickens triggered by a reduced balanced protein diet. Additionally, direct effects of such a diet on liver metabolism was assessed. In the F0 generation, female chickens were fed a C or RP diet, and the progeny was subjected to the same two diets, resulting in four experimental groups, $\mathrm{C} / \mathrm{C}, \mathrm{C} / \mathrm{RP}, \mathrm{RP} / \mathrm{C}$ and $\mathrm{RP} / \mathrm{RP}$. Only female chickens were considered in this study and feed allocation was adapted weekly to maintain a similar body weight. However, when we look at 44 weeks of age, among the 5 chickens we sampled per group, body weights differed with regards to both the F0 and F1 diet, but without interaction effect. The chickens that received a $\mathrm{C} / \mathrm{RP}$ diet had a significantly higher body weight than the $\mathrm{RP} / \mathrm{C}$ chickens, with the $\mathrm{C} / \mathrm{C}$ and RP/RP chickens at intermediate weight. Chickens in this experiment were a subset of those described by Lesuisse et al. (2018a) and the results for proportional breast muscle weight, proportional abdominal fat weight and proportional liver weight did not differ much from those earlier described. In general, it is the F1 feed that influenced these body composition parameters the most. The proportional breast muscle weight was higher in the $\mathrm{C} / \mathrm{C}$ chickens when compared to the $\mathrm{C} / \mathrm{RP}$ and RP/RP chickens, with the RP/C chickens as intermediate. In addition, $\mathrm{C} / \mathrm{RP}$ and $\mathrm{RP} / \mathrm{RP}$ chickens had more abdominal fat compared to $\mathrm{C} / \mathrm{C}$ and $\mathrm{RP} / \mathrm{C}$ chickens, and proportional liver weights were the highest in the C/RP chickens, and were significantly higher when compared to the RP/C chickens. These changes are in accordance with other studies where low and high protein diets were administered while maintaining similar body weights in broiler breeders $^{(27,28)}$.

The liver transcriptome revealed interesting differences when looking at specific group comparisons and examining the genes that were up- and downregulated between these groups of chickens. Gene ontology analyses showed that a direct effect of a control diet $(\mathrm{C} / \mathrm{C}$ versus $\mathrm{C} / \mathrm{RP}$ 
and RP/C versus RP/RP) evoked the molecular function term for transaminase activity, independent of maternal diet. One of the 3 genes in the intersection of DE genes that appeared in these comparisons was the ornithine aminotransferase gene OAT (Table 1). Ornithine is involved in the urea cycle and gets converted by the OAT enzyme into glutamate and proline ${ }^{(29,30)}$. In the liver it has previously been described that the enzymatic activity of OAT proportionally increases with dietary protein levels. This is accomplished by an increase in the number of hepatocytes expressing OAT in chickens fed a high protein diet as compared to those fed a low protein diet ${ }^{(31)}$. In a reduced protein diet, it is seen that nitrogen excretion decreases and $\mathrm{N}$ retention increases ${ }^{(16,32)}$. The rise of ornithine aminotransferase could potentially help with the detoxification of the ammonia excess induced in diets with higher protein levels ${ }^{(33)}$.

The feed formulations for all groups were isoenergetic and the fat percentage was similar in the $\mathrm{C}$ and RP diets, but chickens in the RP groups were fed proportionally more to maintain a similar body weight ${ }^{(15)}$. To keep the diets isoenergetic, protein was substituted by carbohydrates. Combined with the fact that the RP groups were allocated more feed, the RP groups ingested more carbohydrates than the $\mathrm{C}$ groups. However, the feed of the $\mathrm{F} 0$ generation seemed to be the determining factor on which metabolic pathway was mainly triggered. For animals descending from the control F0 hens, a reduced balanced protein diet upregulated the fatty acid metabolism pathway significantly. FADS1, FADS2 and ELOVL2 play a central key in the biosynthesis of long chain n-3 and n-6 polyunsaturated fatty acids (PUFA) ${ }^{(34)}$. FADS1 and FADS2 are necessary to introduce cis double bonds in fatty acids and ELOVL2 catalyzes carbon chain extensions. They convert essential fatty acids such as linoleic acid (LA) and alpha-linolenic acid (ALA) into arachidonic acid (AA), eicosapentaenoic acid (EPA) and docosahexaenoic acid (DHA) ${ }^{(35)}$. FADS1, FADS2 and ELOVL2 were highly expressed and at least 2-fold upregulated in the C/RP group compared to the $\mathrm{C} / \mathrm{C}$ group, but this effect was not observed between $\mathrm{F} 1$ animals on a RP diet versus $\mathrm{C}$ diet descending from F0 hens on a RP diet. Instead, in the liver of animals on a RP diet versus $\mathrm{C}$ diet, descending from the RP F0 hens (RP/RP vs RP/C), an upregulation of fructose and mannose metabolism was seen, with elevated levels of PFKL, PFKFB4 and $A L D O C$. This differential expression due to the RP diet was not observed for animals descending from the control F0 hens (C/RP vs C/C). These genes are key players in the glycolysis and point to more glycogen breakdown as a result of a higher demand of energy ${ }^{(36,37)}$. One of the genes, 
TENM2, found in common between $\mathrm{C} / \mathrm{C}$ vs $\mathrm{C} / \mathrm{RP}$ and $\mathrm{RP} / \mathrm{C}$ vs $\mathrm{RP} / \mathrm{RP}$ comparison, and thus important for a direct reduced protein diet effect regardless of maternal diet, was upregulated in both reduced protein groups (Table 1). Interestingly, TENM2 has been described to be involved in adipogenic differentiation ${ }^{(38)}$. An upregulation of TENM2 in the RP/RP and C/RP groups, when compared to the $\mathrm{RP} / \mathrm{C}$ and $\mathrm{C} / \mathrm{C}$ groups respectively, could be the result of a higher uptake of carbohydrates and fats in the groups reared on a reduced balanced protein diet, but were allocated more feed.

It is very interesting that the greatest number of differentially expressed genes in our comparisons was caused by the maternal diet when chickens were fed an RP diet, i.e. between the $\mathrm{C} / \mathrm{RP}$ and RP/RP groups. This was also shown by the PCA plot that completely separated the $\mathrm{C} / \mathrm{RP}$ and RP/RP groups. Gene ontology analysis of these DE genes exhibited the negative regulation of cellular biosynthetic processes as highest biological process GO term, upregulated in the RP/RP chickens when compared to the C/RP chickens. It seems that the RP/RP animals are having a lower amount of genes expressed, and could therefore potentially show a lower metabolism rate than the $\mathrm{C} / \mathrm{RP}$ animals. Nevertheless, $\mathrm{T}_{3}$ and $\mathrm{T}_{4}$ concentrations in the plasma were not found to be different between these two groups ${ }^{(15)}$. However, in line with a decreased metabolism, Li et al. ${ }^{(39)}$ reported significant differences in behavior due to maternal diet in animals fed a RP diet. RP/RP animals were less active as reflected by a decreased level of litter scratching, reduced feather pecking behavior and an increased time spent sitting compared to the $\mathrm{C} / \mathrm{RP}$ animals. To find the most important drivers behind the differences between $\mathrm{C} / \mathrm{RP}$ and RP/RP animals, we performed a weighted gene correlation network analyses (WGCNA). This resulted in one significant module whose eigengene was higher expressed in the C/RP group compared to the RP/RP group. In the $\mathrm{C} / \mathrm{RP}$ group the GO term 'autophagy' represented an upregulated KEGG pathway compared to the RP/RP group, and the module contained multiple post-translational modification genes, reflected by significant GO terms such as 'phosphorylated amino acid binding'. Hub genes of this module are those with a high gene significance (GS) and high module membership (MM). An example of such a hub gene is the top annotated gene for GS, METTL21C, which encodes for a methyltransferase protein and has also been found to regulate autophagy-associated protein breakdown ${ }^{(40)}$. So, when animals, programmed for a RP 
diet by the F0 generation, are fed a RP diet themselves, they seem to have less protein breakdown compared to animals whose mothers were fed the control diet.

The upregulated autophagic GO term in the WGCNA for the C/RP group versus the RP/RP group reinforces our DE analysis. Upon fasting, it is well-establised that cells shift their metabolism from glucose to fatty-acid oxidation to produce energy and an autophagic degradation of lipid droplets is thought to mobilize triglycerides during the starvation processes $^{(41,42)}$. It seems that the fatty acid metabolism was activated significantly more in the $\mathrm{C} / \mathrm{RP}$ chickens when compared with their control group $\mathrm{C} / \mathrm{C}$, while glucose metabolism took the overhand for RP/RP chickens when compared to their control group C/RP, as described above. This may suggest that the C/RP chickens are in a deeper starvation state than the RP/RP chickens, or, in other words, the RP/RP chickens seemed to be more maternally adapted to the reduced balanced protein diet than the C/RP chickens.

\section{Conclusion}

Irrespective of diets in the F0 generation, chickens reared on a control diet (C) showed an upregulation of transaminase activity in the liver compared to a reduced protein diet (RP), which might indicate a higher amino acid catabolism and uric acid synthesis. However, the F0 diet influenced the metabolism triggered by the reduced balanced protein (RP) diet fed to the F1 generation, being the fatty acid metabolism when the mothers received a $\mathrm{C}$ diet or the glycolysis metabolism when the mothers received a RP diet. It seems as if chickens, when fed a RP diet, act on the reduced amount of protein in the diet either by using their reserves through an autophagic degradation of lipid droplet when the F0 generation was fed a control diet, or by lowering their entire metabolism for those animals descending from RP-fed hens. The distinction between these two coping mechanisms appears to be guided by the maternal diet.

\section{Acknowledgments}

The authors would like to acknowledge the technical assistance performed by Daniel Vermeulen, Astrid Koppenol, An Bautil, Winnie Clímaco, Luís Daniel Giusti Bruno, and André Respen. 


\section{Financial Support}

This work was partly supported by the Agency for Innovation by Science and Technology in Flanders (IWT) Agency for Flanders Innovation \& Entrepreneurship (VLAIO) (Grant no. 141671).

\section{Conflict of Interest}

None

\section{Authorship}

MS wrote the paper. JB conceived and supervised the study. NE, JL and JB developed the experimental design. JL, CLi and SS organized the animal trails and samplings. JL and CLa performed RNA extractions. MS executed DE and GO analyses. JL and MS performed statistical analyses. All authors (MS, JL, CLa, CLi, SS, NE and JB) have read, edited and approved the manuscript. 


\section{References}

1. Hofmann P, Siegert W, Kenéz A et al. (2019). Very low crude protein and varying glycine concentrations in the diet affect growth performance, characteristics of nitrogen excretion, and the blood metabolome of broiler chickens. J Nutr 149, 1122-1132.

2. Hilliar M, Huyen N, Girish CK et al. (2019). Supplementing glycine, serine, and threonine in low protein diets for meat type chickens. Poult Sci 98,6857-6865.

3. Ndazigaruye G, Kim DH, Kang CW et al. (2019). Effects of low-protein diets and exogenous protease on growth performance, carcass traits, intestinal morphology, cecal volatile fatty acids and serum parameters in broilers. Animals (Basel) 9, E226.

4. Jahan-Mihan A, Rodriguez J, Christie C et al. (2015). The role of maternal dietary proteins in development of metabolic syndrome in offspring. Nutrients 7, 9185-9217.

5. Moraes TGV, Pishnamazi A, Wenger II et al. (2019). Energy and protein dilution in broiler breeder pullet diets reduced offspring body weight and yield. Poult Sci 98, 2555-2561.

6. Lv Z, Fan H, Song B et al. (2019). Supplementing genistein for breeder hens alters the fatty acid metabolism and growth performance of offsprings by epigenetic modification. Oxid Med Cell Longev 9214209.

7. Rao K, Xie J, Yang X et al. (2009). Maternal low-protein diet programmes offspring growth in association with alterations in yolk leptin deposition and gene expression in yolk-sac membrane, hypothalamus and muscle of developing Langshan chicken embryos. Br J Nutr 102, 848-857.

8. Zhu YW, Li WX, Lu L et al. (2017). Impact of maternal heat stress in conjunction with dietary zinc supplementation on hatchability, embryonic development, and growth performance in offspring broilers. Poult Sci 96: 2351-2359.

9. Beckford RC, Howard SJ, Das S et al. (2017). Maternal consumption of fish oil programs reduced adiposity in broiler chicks. Sci Rep 7, 13129.

10. $\mathrm{Hu} \mathrm{Y}$, Sun Q, Hu Y et al. (2018). Corticosterone-induced lipogenesis activation and lipophagy inhibition in chicken liver are alleviated by maternal betaine supplementation. $J$ Nutr 148, 316-325.

11. Aiqueperse N, Calandreau L, Bertin A (2013). Maternal diet influences offspring feeding behavior and fearfulness in the precocial chicken. PLoS One 8, e77583. 
12. de Haas EN, Calandreau L, Baéza E et al. (2017) Lipids in maternal diet influence yolk hormone levels and post-hatch neophobia in the domestic chick. Dev Psychobiol 59, 400-409.

13. Van der Waaij EH, van den Brand H, van Arendonk JA et al. (2011). Effect of match or mismatch of maternal-offspring nutritional environment on the development of offspring in broiler chickens. Animal 5:741-748.

14. Lesuisse J, Li C, Schallier S et al. (2017). Feeding broiler breeders a reduced balanced protein diet during the rearing and laying period impairs reproductive performance but enhances broiler offspring performance. Poult Sci 96, 3949-3959.

15. Lesuisse J, Li C, Schallier S, et al. (2018a). Multigenerational effects of a reduced balanced protein diet during the rearing and laying period of broiler breeders. 1. Performance of the F1 breeder generation. Poult Sci 97, 1651-1665.

16. Lesuisse J, Schallier S, Li C et al. (2018b). Multigenerational effects of a reduced balanced protein diet during the rearing and laying period of broiler breeders. 2. Zootechnical performance of the F1 broiler offspring. Poult Sci 97, 1666-1676.

17. Aviagen (2013). Ross 308 Parent Stock Management Guide. Aviagen Incorporated, Huntsville, AL.

18. Willson NL, Forder REA, Tearle R et al. (2018). Transcriptional analysis of liver from chickens with fast (meat bird), moderate (F1 layer x meat bird cross) and low (layer bird) growth potential. BMC Genomics 19, 309.

19. Khalique A, Zeng D, Wang H et al. (2019). Transcriptome analysis revealed ameliorative effect of probiotic Lactobacillus johnsonii BS15 against subclinical necrotic enteritis induced hepatic inflammation in broilers. Microb Pathog 132, 201-207.

20. Andrew S (2010). FastQC: a quality control tool for high throughput sequence data. http://www.bioinformatics.babraham.ac.uk/projects/fastqc. (accessed September 2019).

21. Trapnell C, Roberts A, Goff L et al. (2012). Differential gene and transcript expression analysis of RNA-seq experiments with TopHat and Cufflinks. Nat protoc 7, 562-578.

22. Love MI, Huber W, Anders S (2014). Moderated estimation of fold change and dispersion for RNA-seq data with DESeq2. Genome Biology 15, 550.

23. Oliveros JC (2007). Venny. An interactive tool for comparing lists with Venn's diagrams. http://bioinfogp.cnb.csic.es/tools/venny/index.html. (accessed October 2019). 
24. Reimand J, Kull M, Peterson H et al. (2007). g:Profiler - a web-based toolset for functional profiling of gene lists from large-scale experiments. Nucleic Acids Research 35, W193-W200. 25. Langfelder P, Horvath S (2008). WGCNA: An R package for weighted correlation network analysis. BMC Bioinformatics 9:559.

26. Bayen S, Saini S, Gaur P et al. (2018). PRMT1 promotes hyperglycemia in a FoxO1dependent manner, affecting glucose metabolism, during hypobaric hypoxia exposure, in rat model. Endocrine 59, 151-163.

27. van Emous RA, Kwakkel RP, van Krimpen MM et al. (2013). Effects of growth patterns and dietary crude protein levels during rearing on body composition and performance in broiler breeder females during the rearing and laying period. Poultry Science 92, 2091-2100.

28. van Emous RA, Kwakkel RP, van Krimpen MM et al. (2015). Effects of dietary protein levels during rearing and dietary energy levels during lay on body composition and reproduction in broiler breeder females. Poultry Science 94, 1030-1042.

29. Ginguay A, Cynober L, Curis E et al. (2017). Ornithine aminotransferase, an important glutamate-metabolizing enzyme at the crossroads of multiple metabolic pathways. Biology (Basel) 6, 18.

30. Sivashanmugam M, Jaidev J, Umashankar V et al. (2017). Ornithine and its role in metabolic diseases: an appraisal. Biomed Pharmacother 86, 185-194.

31. Matsuzawa T, Kobayashi T, Tashiro K et al. (1994). Changes in ornithine metabolic enzymes induced by dietary protein in small intestine and liver: intestine-liver relationship in ornithine supply to liver. J Biochem 116,721-727.

32. Gou ZY, Jiang SQ, Jiang ZY et al. (2016). Effects of high peanut meal with different crude protein level supplemented with amino acids on performance, carcass traits and nitrogen retention of Chinese Yellow broiler. J Anim Physiol Anim Nutr 100, 657-664.

33. Boon L, Geerts WJ, Jonker A et al. (1999). High protein diet induces pericentral glutamate dehydrogenase and ornithine aminotransferase to provide sufficient glutamate for pericentral detoxification of ammonia in rat liver lobules. Histochem Cell Biol 111, 445-452.

34. Jing M, Gakhar N, Gibson RA et al. (2013). Dietary and ontogenic regulation of fatty acid desaturase and elongase expression in broiler chickens. Prostaglandins Leukot Essent Fatty Acids 89, 107-113. 
35. Sun C, Zou M, Wang X et al. (2018). FADS1-FADS2 and ELOVL2 gene polymorphisms in susceptibility to autism spectrum disorders in Chinese children. BMC Psychiatry 18, 283.

36. Kuo T, McQueen A, Chen $\mathrm{T}$ et al. (2015). Regulation of glucose homeostasis by glucocorticoids. Adv Exp Med Biol 872, 99-126.

37. Webb BA, Dosey AM, Wittmann T et al. (2017). The glycolytic enzyme phosphofructokinase-1 assembles into filaments. J Cell Biol 216, 2305-2313.

38. Tews D, Fromme T, Keuper M et al. (2017). Teneurin-2 (TENM2) deficiency induces UCP1 expression in differentiating human fat cells. Mol Cell Endocrinol 443, 106-113.

39. Li C, Hu Q, Lesuisse J et al. (2019). The effect of reduced balanced protein diet on the behavior of female broiler breeders in 2 generations. Poult Sci 98, 4301-4312.

40. Wiederstein JL, Nolte H, Günther S et al. (2018). Skeletal muscle-specific methyltransferase METTL21C trimethylates p97 and regulates autophagy-associated protein breakdown. Cell Rep 23, 1342-1356.

41. Singh R, Kaushik S, Wang Y et al. (2009). Autophagy regulates lipid metabolism. Nature 458, 1131-1135.

42. Saito T, Kuma A, Sugiura Y et al. (2019). Autophagy regulates lipid metabolism through selective turnover of NCoR1. Nat Commun 10, 1567. 


\section{Legends of figures}

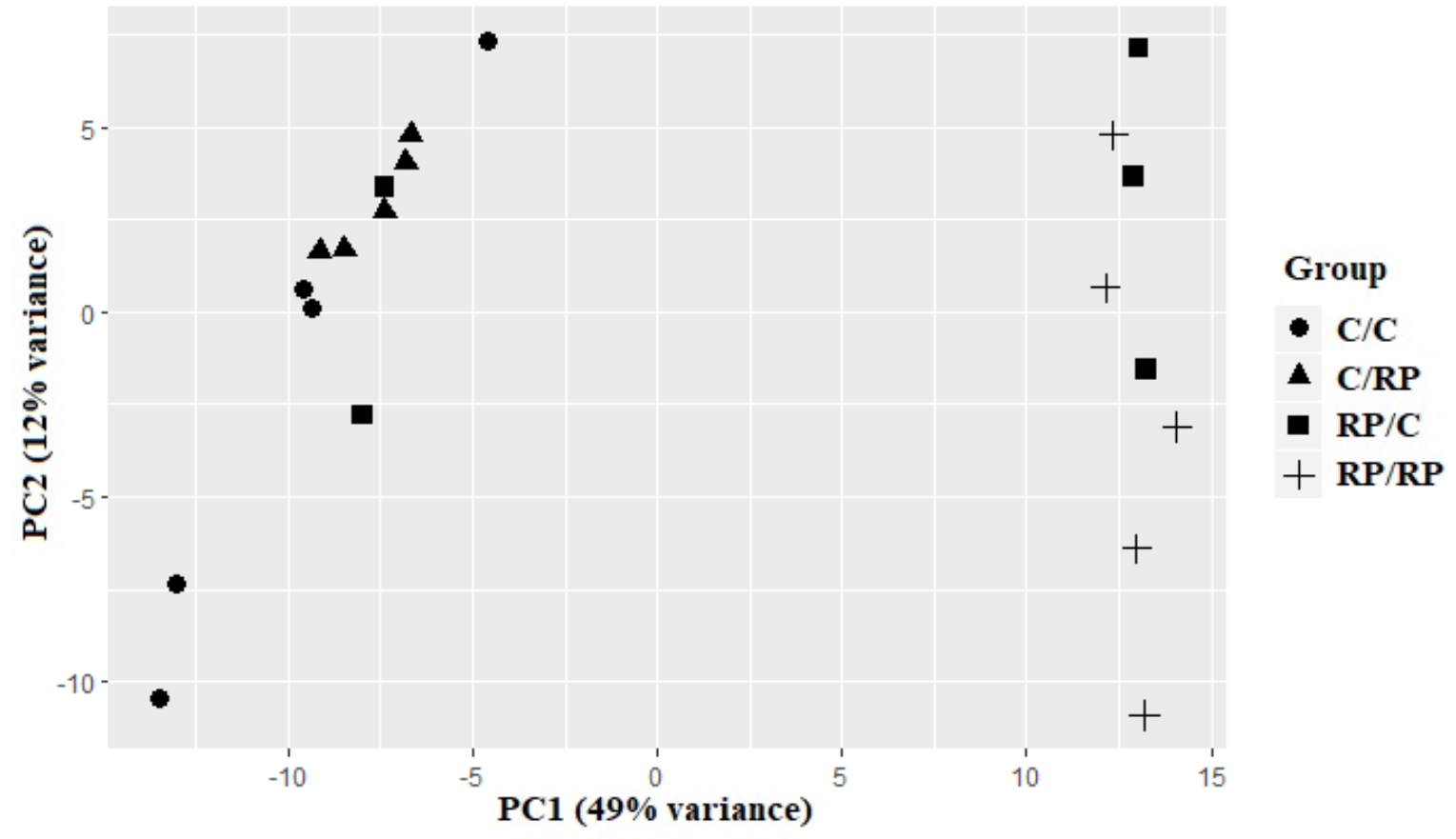

Figure 1. Principal Component Analysis (PCA) plot of the liver RNA-seq data. Only the first two principal components are shown. C/C (circle), C/RP (triangle), RP/C (square) and RP/RP (plus), with $\mathrm{C}$ for control diet and RP for reduced balance protein diet for the diet in respectively the F0 and F1 generation. Letters indicating the breeder feed in respectively the F0 and F1 generation, $\mathrm{C}=\mathrm{Control}$ diet, $\mathrm{RP}=$ Reduced balanced protein diet. Dots represent individuals. 
Accepted manuscript

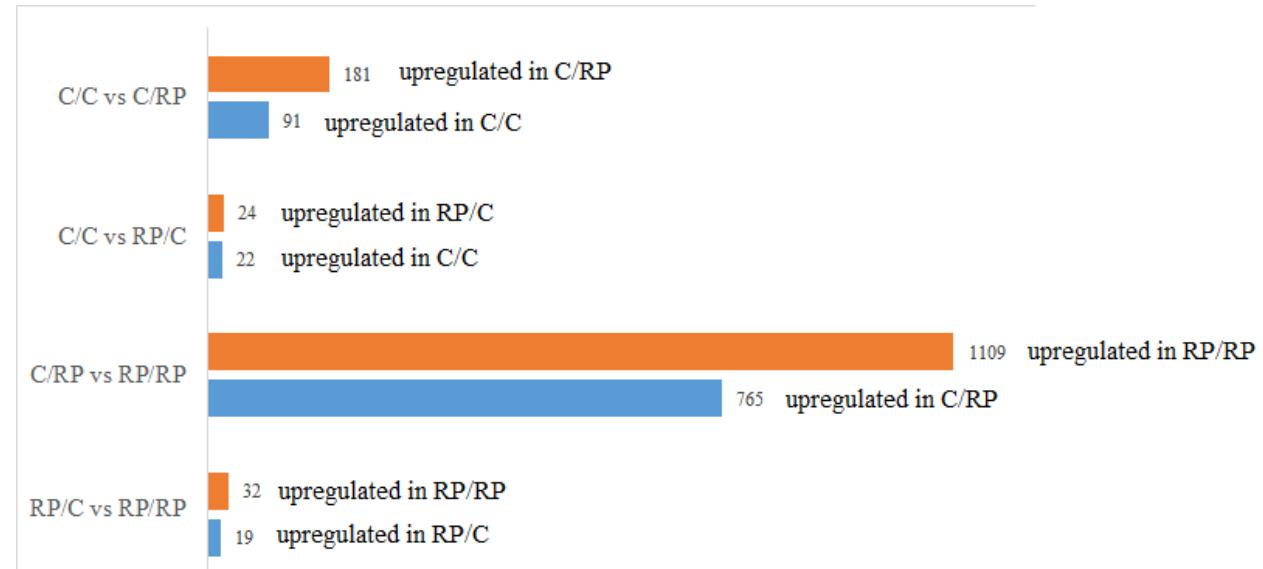

Figure 2. Number of Differential Expressed (DE) genes when comparing the $\mathrm{C} / \mathrm{C}$ with $\mathrm{C} / \mathrm{RP}$ group, the $\mathrm{C} / \mathrm{C}$ with $\mathrm{RP} / \mathrm{C}$ group, the $\mathrm{C} / \mathrm{RP}$ with $\mathrm{RP} / \mathrm{RP}$ group and the $\mathrm{RP} / \mathrm{C}$ with $\mathrm{RP} / \mathrm{RP}$ groups. Number of DE genes is split into those up- and downregulated between the groups compared. Letters indicating the breeder feed in respectively the F0 and F1 generation, $\mathrm{C}=\mathrm{Control}$ diet, $\mathrm{RP}$ $=$ Reduced balanced protein diet. Benjamini-Hochberg correction for multiple testing was set to $\mathrm{FDR}<0.05$. 


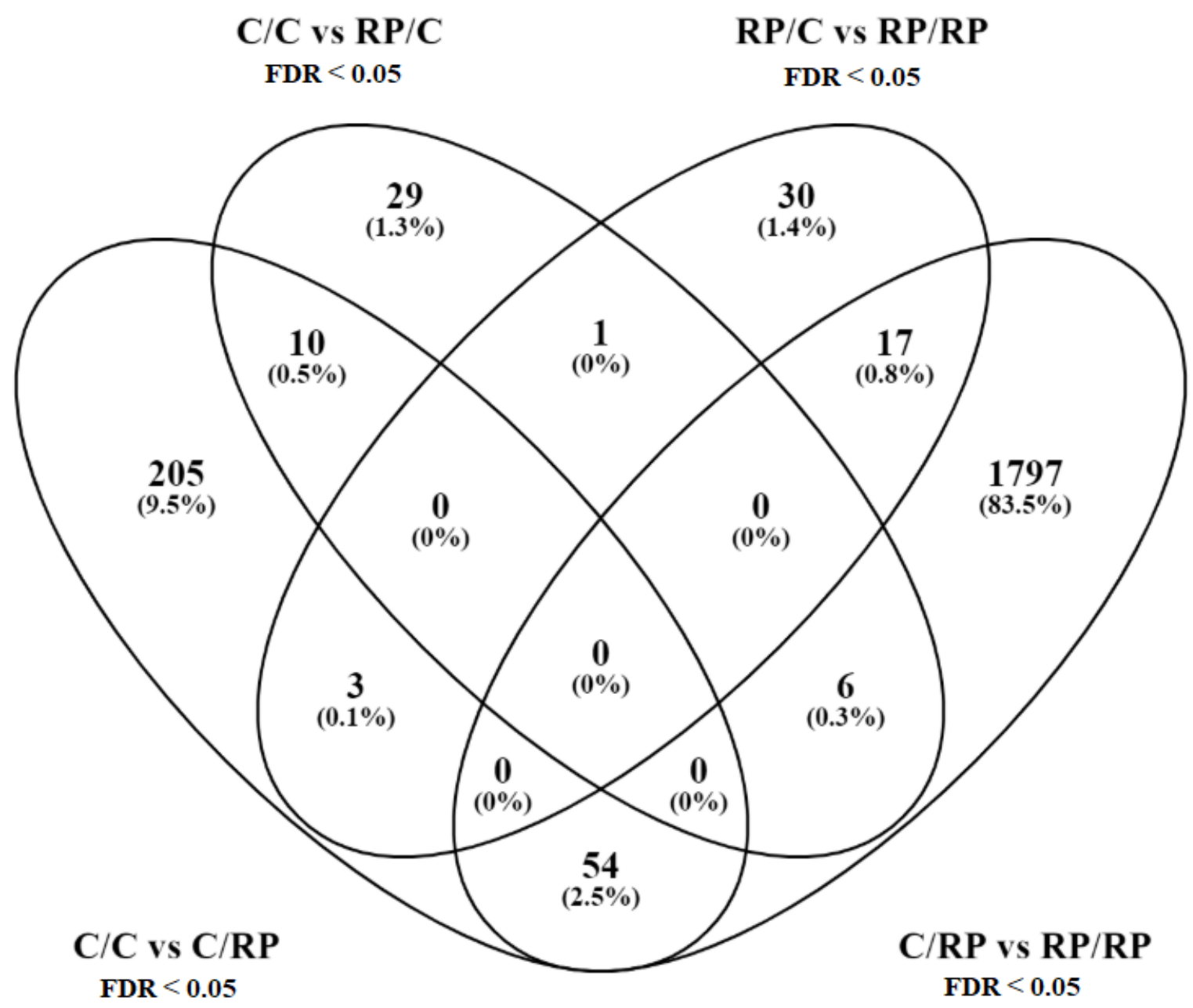

Figure 3. Venn diagram of the number of differentially expressed (DE) genes within different comparisons. Comparisons of interest where those between the groups of which the F0 diet was kept constant, but the F1 diet changed (C/C vs $\mathrm{C} / \mathrm{RP}$ and $\mathrm{RP} / \mathrm{C}$ vs $\mathrm{RP} / \mathrm{RP})$ or vice versa (C/C vs $\mathrm{RP} / \mathrm{C}$ and $\mathrm{C} / \mathrm{RP}$ vs RP/RP). Percentage is given as the percentage of all DE genes. Letters indicating the breeder feed in respectively the F0 and F1 generation, $\mathrm{C}=$ Control diet, $\mathrm{RP}=$ Reduced balanced protein diet. Benjamini-Hochberg correction for multiple testing was set to FDR $<0.05$. 
A

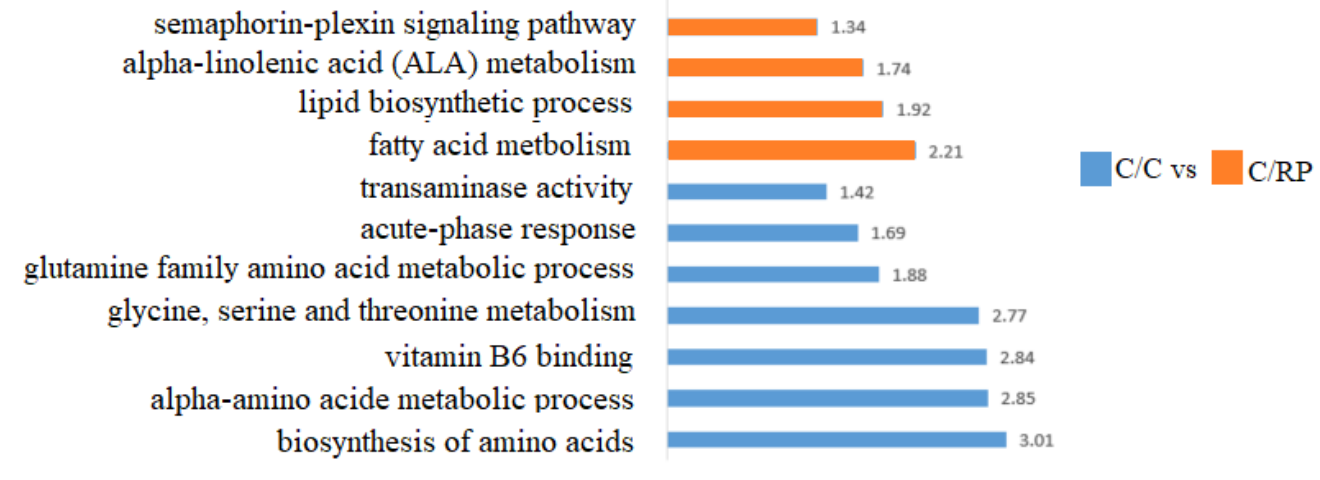

B

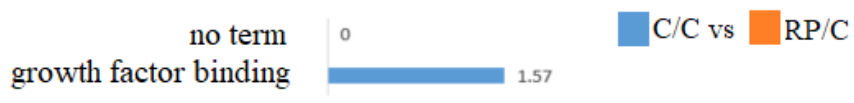

C

negative regulation of gene expression negative regulation of cellular biosynthetic process nucleocytoplasmic carrier activity

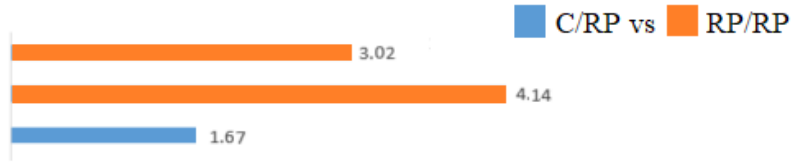

D

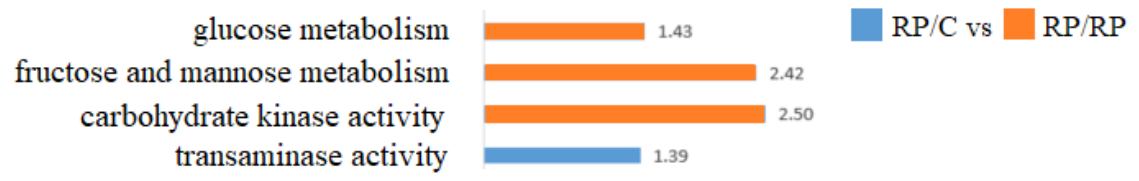

Figure 4. Gene Ontology (GO) analyses results of the contrast between the $\mathrm{C} / \mathrm{C}$ and $\mathrm{C} / \mathrm{RP}$ group (A), the $\mathrm{C} / \mathrm{C}$ and $\mathrm{RP} / \mathrm{C}$ group (B), the $\mathrm{C} / \mathrm{RP}$ and RP/RP group (C) and the RP/C with RP/RP groups (D). Enrichment Score reflected in this figure is the $-\log _{10}$ of the adjusted p-value of the enrichment analysis. Letters indicating the breeder feed in, respectively the F0 and F1 generation, $\mathrm{C}=\mathrm{Control}$ diet, $\mathrm{RP}=$ Reduced balanced protein diet. 


\section{Legends of Tables and Supplementary Tables}

Table 1. Overview of genes that were common $\mathrm{DE}$ genes when comparing $\mathrm{C} / \mathrm{C}$ vs $\mathrm{C} / \mathrm{RP}$ and $\mathrm{RP} / \mathrm{C}$ vs RP/RP, which reflects genes whose expression is affected by giving a direct reduced protein diet, and genes that were common $\mathrm{DE}$ genes when comparing $\mathrm{C} / \mathrm{C}$ vs $\mathrm{RP} / \mathrm{C}$ and $\mathrm{C} / \mathrm{RP}$ vs $\mathrm{RP} / \mathrm{RP}$, which reflects genes whose expression is affected by giving a maternal reduced protein diet. ENSEMBL gene IDs and gene symbols (when available) are given, as well as their binary logarithmic fold change $(\log 2 \mathrm{FC})$ difference and the adjusted p-values for the comparisons of interest.

\begin{tabular}{|c|c|cc|cc|}
\hline \multicolumn{2}{|c|}{ in common between C/C vs C/RP and RP/C vs RP/RP (= direct reduced protein effect) } \\
\hline \multirow{2}{*}{ ENSEMBL gene ID } & \multirow{2}{*}{ Gene Symbol } & \multicolumn{2}{c|}{ C/C vs C/RP } & \multicolumn{2}{c|}{ RP/C vs RP/RP } \\
& & log2FC & adj p-value & log2FC & adj p-value \\
\hline ENSGALG00000001768 & TENM2 & 1.59 & 0.01 & 1.51 & 0.002 \\
ENSGALG00000031158 & OAT & -1.14 & 0.0002 & -1.34 & 0.02 \\
ENSGALG00000038217 & CD82 & -0.47 & 0.04 & -0.46 & 0.05 \\
\hline
\end{tabular}

\begin{tabular}{|c|c|cc|cc|}
\hline \multicolumn{2}{|c|}{ in common between C/C vs RP/C and C/RP vs RP/RP (= maternal reduced protein effect) } \\
\hline \multirow{2}{*}{ ENSEMBL gene ID } & \multirow{2}{*}{ Gene Symbol } & \multicolumn{2}{|c|}{ C/C vs RP/C } & \multicolumn{2}{c|}{ C/RP vs RP/RP } \\
& & log2FC & adj p-value & log2FC & adj p-value \\
\hline ENSGALG00000033682 & - & 1.24 & 0.02 & 2.12 & $3.61 \mathrm{E}-18$ \\
ENSGALG00000037088 & - & 1.22 & 0.04 & 1.07 & $4.36 \mathrm{E}-13$ \\
ENSGALG00000039384 & - & -1.57 & 0.03 & -1.50 & 0.001 \\
ENSGALG00000040350 & - & -1.92 & 0.05 & -2.43 & 0.03 \\
ENSGALG00000041747 & - & -1.61 & 0.05 & -1.13 & 0.04 \\
ENSGALG00000043329 & PTMA & -1.14 & 0.004 & -0.75 & 0.003 \\
\hline
\end{tabular}


Supplementary Table 1. Total weight, body composition (breast muscle (BM) and abdominal fat pad (AF)) and organ weights (liver, pancreas, oviduct, ovarium) of $\mathrm{C} / \mathrm{C}, \mathrm{C} / \mathrm{RP}, \mathrm{RP} / \mathrm{C}$, and $\mathrm{RP} / \mathrm{RP}$ breeders (letters indicating the breeder feed in, respectively, the F0 and F1 generations; C $=$ Control diet, $\mathrm{RP}=$ Reduced balanced protein diet $)$ at 44 weeks of age $(n=5)$. Data are presented as mean \pm SEM. ${ }^{\mathrm{A}, \mathrm{B}}$ : different superscript means significant difference for $\mathrm{F} 0 \operatorname{diet}(\mathbf{p}<\mathbf{0 . 0 5}){ }^{\mathrm{x}, \mathrm{y}}$ : different superscript means significant difference for F1 diet $(\mathbf{p}<\mathbf{0 . 0 5})$.

Supplementary Table 2. Overview of the members of the significant module $(p<0.001)$ resulted from the WGCNA on the C/RP versus RP/RP comparison. The module was correlated with the treatment at a correlation of -0.88 . Since $\mathrm{C} / \mathrm{RP}$ was set to 0 and RP/RP to 1 , this means that the overall expression was higher in the $\mathrm{C} / \mathrm{RP}$ group then in the RP/RP group. Genes shown are given by ENSEMBL gene IDs, and whenever available ENSEMBL protein ID and Symbol name are given as well. GS represents gene significance, or how well does this gene relate to the difference in treatment, which is here the maternal feed. p.GS is the p-value for GS. MM represents module membership, or how well does this gene fit in this module. P.MM is the pvalue for MM.

Supplementary Table 3. Overview of Gene ontology (GO) terms up- and downregulated when comparing the $\mathrm{C} / \mathrm{C}$ and $\mathrm{C} / \mathrm{RP}$ group, the $\mathrm{C} / \mathrm{C}$ and $\mathrm{RP} / \mathrm{C}$ group, the $\mathrm{C} / \mathrm{RP}$ and $\mathrm{RP} / \mathrm{RP}$ group and the RP/C with RP/RP group, as well as those for the significant module found by WGCNA whose members are shown in Supplementary Table 2. The tool used was g:profiler, and Gallus gallus as organism. GO terms are subdivided in molecular function (GO:MF), biological process (GO:BP) and cellular component (GO:CC) level, or according to KEGG or Reactome (REAC) database. Adjusted p-values, $-\log _{10}$ of the adjusted p-values as well as Term size, Query size and their overlap (Intersection) are shown. Genes of the intersection are represented as ENSEMBL gene IDs. 